\title{
蒙特卡罗法估算真实溶液的无限稀释活度系数 *
}

\section{鲍坚斌 韩世钧}

(浙江大学化学系, 杭州 310027)

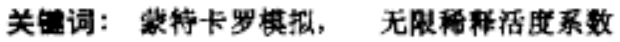

在无限稀释活度系数实验测定、模型预测等方面的研究工作不断深入的同时，基于系统 的微观分子结构信息通过计算机分子模拟方法来求取无限稀释活度系数的研究也更多地开展起 来 ${ }^{[1,2]}$, 但是鉴于嫡类热力学性质, 如自由能、化学位等的特殊性, 开展的模侦工作总的说来是 初步的. 许多方法复杂, 实现的难度较大, 而研究的系统较多地针对于模型流体混合物 (model fluid mixtures). 本文是蒙特卡罗法估算真实溶液的无限稀释活度系数的一个尝试, 研究对象选 取 $298.15 \mathrm{~K}$ 温度条件下甲醇的水溶液.

\section{1 原理}

对于一个 $N T V$ 系综, 化学位定义为

$$
\begin{aligned}
\mu & =\left(\frac{\partial A}{\partial N}\right)_{T, V} \\
& =\lim _{\mathrm{d} N \rightarrow 0} \frac{A(N)-A(N-\mathrm{d} N)}{\mathrm{d} N} \\
& \simeq A(N)-A(N-1)
\end{aligned}
$$

由于

$$
\begin{aligned}
& A(N)=-k T \ln \left[\frac{Q_{N}}{N ! \Lambda^{3 N}}\right] \\
& =-k T \ln Q_{N}+k T \ln N !+N k T \ln \Lambda^{3} \\
& A(N-1)=-k T \ln Q_{N-1}+k T \ln (N-1) !+(N-1) k T \ln \Lambda^{3}
\end{aligned}
$$

其中

$$
\begin{aligned}
& Q_{N}=\int \cdots \int \mathrm{d} \boldsymbol{R}_{1} \mathrm{~d} \boldsymbol{R}_{2} \cdots \mathrm{d} \boldsymbol{R}_{N-1} \mathrm{~d} \boldsymbol{R}_{N} \exp \left(-\beta U_{N}\right) \\
& Q_{N-1}=\int \cdots \int \mathrm{d} \boldsymbol{R}_{1} \mathrm{~d} \boldsymbol{R}_{2} \cdots \mathrm{d} \boldsymbol{R}_{N-1} \exp \left(-\beta U_{N-1}\right)
\end{aligned}
$$

可以将 $U_{N-1}$ 看成:

$$
U_{N-1}\left(\boldsymbol{R}_{1}, \boldsymbol{R}_{2}, \cdots, \boldsymbol{R}_{N-1}\right)=U_{N}\left(\boldsymbol{R}_{1}, \boldsymbol{R}_{2}, \cdots, \boldsymbol{R}_{N-1}, \boldsymbol{R}_{N}\right)-\sum_{i=1}^{N-1} U\left(\boldsymbol{R}_{i}, \boldsymbol{R}_{N}\right)
$$

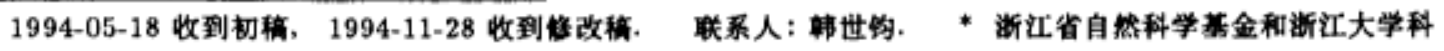
学基金算助项且 
论

$$
B\left(\boldsymbol{R}_{1}, \boldsymbol{R}_{2}, \cdots, \boldsymbol{R}_{N-1}, \boldsymbol{R}_{N}\right)=\sum_{i=1}^{N-1} U\left(\boldsymbol{R}_{i}, \boldsymbol{R}_{N}\right)
$$

综合式 (1) (7) 有:

$$
\begin{aligned}
& \beta \mu=\ln \left(N A^{3}\right)-\ln \left[\frac{\left.\int \cdots \int \mathrm{d} \boldsymbol{R}_{1 \mathrm{~d}} \boldsymbol{R}_{2 \cdots \mathrm{d}} \boldsymbol{R}_{N-1} \mathrm{~d} \boldsymbol{R}_{N \exp \left(-\beta U_{N}\right)}\right] \iint \mathrm{d} \boldsymbol{R}_{1 \mathrm{~d}} \boldsymbol{R}_{2 \cdots \mathrm{d}} \boldsymbol{R}_{N-1} \exp \left(-\beta U_{N}\right) \exp (\beta B)}{]}\right] \\
& =\ln \left(\rho A^{3}\right)+\ln \left[\frac{\int \cdots \int \mathrm{d} \boldsymbol{R}_{1} \mathrm{~d} \boldsymbol{R}_{2} \cdots \mathrm{d} \boldsymbol{R}_{N-1} \mathrm{~d} \boldsymbol{R}_{N} \exp \left(-\beta U_{N}\right) \exp (\beta B)}{\int \cdots \int \mathrm{d} \boldsymbol{R}_{1} \mathrm{~d} \boldsymbol{R}_{2} \cdots \mathrm{d} \boldsymbol{R}_{N-1} \mathrm{~d} \boldsymbol{R}_{N} \exp \left(-\beta U_{N}\right)}\right] \\
& =\ln \left(\rho A^{3}\right)+\ln <\exp (\beta B)>_{N}
\end{aligned}
$$

或者

$$
\beta \mu^{r}=\ln \langle\exp (\beta B)\rangle_{\mathrm{N}}
$$

其中 $\mu^{r}=\mu-\mu^{i-g}, \rho=N / V, \beta=(k T)^{-1},\langle\cdots\rangle_{N}$ 为系综平均.

对丁纯㴒质 $B$, 式 (9) 为:

$$
\left.\beta_{\mu^{r^{*}}}=\ln <\exp \left[\beta B\left(\boldsymbol{R}_{B_{1}}, \boldsymbol{R}_{B_{2}}, \cdots, \boldsymbol{R}_{B_{N-1}}, \boldsymbol{R}_{B_{N}}\right)\right]\right\rangle_{N}
$$

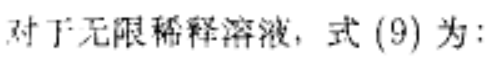

$$
\beta \mu^{r^{x}}=\ln <\exp \left[\beta B\left(\boldsymbol{R}_{A_{1}}, \boldsymbol{R}_{A_{2}}, \cdots, \boldsymbol{R}_{A_{X^{\prime}-1}}, \boldsymbol{R}_{B_{N}}\right)\right]>_{N}
$$

因此无限稀释活度系数 $\gamma_{B(A)}^{\infty}$ 为:

$$
\begin{aligned}
\gamma_{B(A)}^{\infty}= & \exp \left(\beta \mu^{r^{\infty}}-\beta \mu^{r^{*}}\right) \\
= & \frac{\left\langle\exp \left[\beta B\left(\boldsymbol{R}_{A_{1}}, \boldsymbol{R}_{A_{2}}, \cdots, \boldsymbol{R}_{A_{N-1}}, \boldsymbol{R}_{B_{N}}\right)\right]\right\rangle_{N}}{\left\langle\exp \left[\beta B\left(\boldsymbol{R}_{B_{1}}, \boldsymbol{R}_{B_{2}}, \cdots, \boldsymbol{R}_{B_{N-1}}, \boldsymbol{R}_{B_{N}}\right)\right]\right\rangle_{N}}
\end{aligned}
$$

由式 (12) 可以知道, 通过蒙特卡罗多法计算 1 个济质分子 (常称之为 “测试粒子”) 与 $N-1$ 个搳剂分子或溶质分子 (通过周期性边界条件展开) 的作用能之和的系综平均可以求出无限稀释 活度系数. 这种方法针对的实际过程相览于将测试粒子移除系综的过程, 故可称之为 “移除粒子 法”, 显然这比常规的 “测试䊉子法”[2] 更易于实现, 尤适于高密度的昼实济䍇系统的模拟, 而且 核法往往可以与系统的力学性质如内能、热容等同时进行模拟.

\section{2 模拟细节}

围禀的水济被是个包含有偶极的真实系统, 其分子间势能函数选取 Jorgensen ${ }^{[3.4]}$ 的通用 分于伍能函数 (transferable intermolecular potential function, TIP). 它将甲音、水分子各分 为 3 个基团. 甲分子分为 $\mathrm{H} 、 \mathrm{O} 、 \mathrm{CH}_{3}$, 水分子分为 $\mathrm{H} 、 \mathrm{O} 、 \mathrm{H}$, 仿能函数的形式为:

$$
U_{A B}=\sum_{a \in \in A} \sum_{b \in B}\left(\frac{q_{a} q_{b} e^{2}}{r_{a b}}+\frac{A_{a} A_{b}}{r_{a b b}^{12}}-\frac{C_{a} C_{b}}{r_{a b}^{6}}\right)
$$


方程的多数 $q 、 A 、 C$ 见文献 $[3,4]$, 分子尺寸见參考文献 $[5]$.

容特卡罗模证程序以 FORTRAN 语言自行编制, 可在 HP720 计算机上运行. 每次模拟 取 108 个分子, 其中 1 个为㵏质分子, 余下为溶剂或溶质分子. 以面心立方晶格为起始构型,

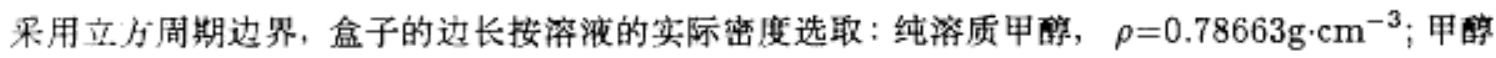
在水中的无限稀释淮济， $\rho \approx 1 \mathrm{~g} \cdot \mathrm{cm}^{-3}$. 模拟采用球形 “割去法 (cutoff method)”, 截断半径为 盒子边长的一半, 载㽤距离之外的分子间作用能采用文献 $[3,4]$ 的方法校正. 在模拟过程中, 考 哕分子的三维平动和三维转动, 初始平动范围为 $\pm 0.05 \AA$, 转动角度范围为 $\pm 5^{\circ}$, 在模拟过程中 作一定调整, 使分子运动接受率接近 $50 \%$ 。每次模拟生成 $1.5 \times 10^{6}$ 个构型, 前 $1 \times 10^{6}$ 个产生平 衡构型, 后 $0.5 \times 10^{6}$ 个用于统计.

表 1 慗特卡罗模拟结果

Table 1 Monte Carlo simulation results

\begin{tabular}{|c|c|c|c|c|c|c|}
\hline \multirow[b]{2}{*}{ system } & \multicolumn{2}{|c|}{$\Delta_{v} H_{m} / \mathrm{kJ} \cdot \mathrm{mol}^{-1}$} & \multicolumn{2}{|c|}{$C_{V, m} / \mathrm{J} \cdot \mathrm{mol}^{-1} \cdot \mathrm{K}^{-1}$} & \multicolumn{2}{|c|}{$\gamma^{\infty}$} \\
\hline & this work & literature & this work & literature & this work & literature \\
\hline \multirow[t]{2}{*}{ methanol } & 32.6 & $32.6^{\mathrm{a}}$ & 69.0 & $68.2^{\mathrm{a}}$ & & \\
\hline & & $32.2^{\mathrm{b}}$ & & $71.1^{\mathrm{b}}$ & & \\
\hline dilute solution & 37.7 & $38.5^{\mathrm{b}}$ & 62.8 & $63.6^{\mathrm{b}}$ & 1.32 & $1.65^{c}$ \\
\hline
\end{tabular}

a-simulation values, ref. $[3,4]$; b-simulation values, ref. [5];

c-experimental value, ref. [6]

\section{3 结果与讨论}

模找得到的 $298.15 \mathrm{~K}$ 温度条件下甲琮在水中的无限稀释活度系数, 和 2 个相关系统 (纯甲

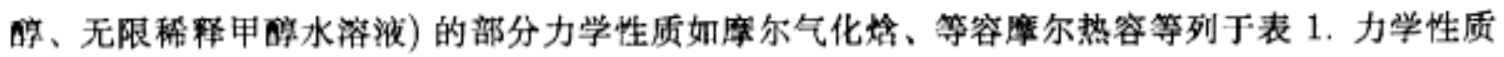
与文献值的对比结果表明, 我们编制的蒙特卡罗模拟程序是可靠的. $\gamma^{\infty}$ 的估第结果为 1.32 , 与 实哈测定值 $1.65^{[6]}$ 虽然有不少差频, 但却是合理的. 分析误差产生的原因, 至少包括了: 一是模 拟方法的取样空间过于集中在低能区. 虽然通过两个系综平均值的相比可部分地弥补这一点, 使 无限稀释活度系数比化学位有了更高的精度. 二是对偶势能 (pairwise potential energy) 函数 TIP 的局限性. 文献 $[3,4]$ 认为对于甲酸 - 水系统, 三体作用可达到 $15 \%$ 左右, 所幸的是, 本 文的模拟方法可在较大程度上消去其部分的影响. 另外, TIP 仅为一种简单的对偶势, 更完备 的势函数将有利于模拟精度的提高.

式 (8) 中的系综平均 $\left\langle\exp [\beta B]>_{N}\right.$ 可以进一步写为:

$$
\begin{gathered}
<\exp \left[\beta B\left(\boldsymbol{R}_{1}, \boldsymbol{R}_{2}, \cdots, \boldsymbol{R}_{N-1}, \boldsymbol{R}_{N}\right)\right]>_{N}=\left\langle\exp \left[\beta \sum_{i=1}^{N-1} U\left(\boldsymbol{R}_{i}, \boldsymbol{R}_{N}\right)\right]>_{N}\right. \\
=<I_{i=1}^{N-1} \exp \left[\beta U\left(\boldsymbol{R}_{i}, \boldsymbol{R}_{N}\right)\right]>_{N}
\end{gathered}
$$

显然这是一个有别丁力学性质的方, 它不是分子对偶性质的加和, 而是其乘积, 从而使得 化学住不可能象力学性质那样表示为一个包含对偶性质的简单积分, 这也正是嫡类热力学性质 模拟的国难所左. 


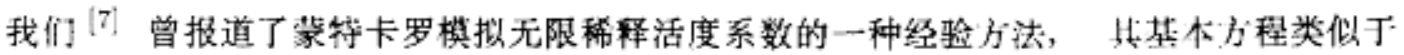
Arrhenius $f$ 程:

$$
a=Z \exp \left[\beta<E>_{N}\right]
$$

其中 $a$ 表示单个溶质分子的活度， $Z$ 为指前因子, $\langle E\rangle_{N}$ 为潧质分子与其周围环境分子的 相互作用能. 如果假设指前因子 $Z$ 仅与溶质分子相关, 则流质分子在不同环境 (溶剂分子 $A$ 或 溶质分子 $B$ ) 中的活度比即为无限稀释活度系数:

$$
\gamma_{B(A)}^{\infty}=\frac{a_{B(A)}}{a_{B(B)}}=\frac{\exp \left[\beta<E_{B(A)}>_{N}\right]}{\exp \left[\beta<E_{B(B)}>_{N}\right]}
$$

其结果与式 (12) 柏一致.

本文作为真实㴒液系统的无限稀释活度系数模拟的一个品试, 其实用性需有更多的实践检 倠.

\title{
考文 献
}

1 Widom B. J. Chem. Phys., 1963, 39:2808

2 Romano S, Singer K. Mol. Phys, 1979, 37:1765

3 Jorgensen W L. J. Am. Chem. Soc., 1981, 103:335

4 Jorgensen W L. J. Am. Chem. Soc., 1981, 103:341

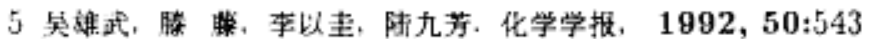

6 Lebert A. Richon D. J. Agric. Food Chem., 1984, 32:1156

7 Bao .J. Chen J, Cai W, et al. Proceedings of 34th IUPAC Congress, Beijing. 1993. 907

\section{A Method for the Estimation of Infinite Dilution Activity Coefficient for a Real Solution by Monte Carlo Simulation}

Bao Jianbin Han Shijun

(Department of Chemistry, Zhejiang University, Hangzho1 310027)

\begin{abstract}
A new method for the estimation of infinite dilution activity coefficient, the removal test-particle method, is proposed and shown in a more practical way for a real solution. By this method, the limiting value of methanol in aqueous solution at $298.15 \mathrm{~K}$ has been studied.
\end{abstract}

Keywords: Monte Carlo simulation, Infinite dilution activity coefficient 